\title{
Memristor-Based Canonical Chua's Circuit: Extreme Multistability in Voltage-Current Domain and Its Controllability in Flux-Charge Domain
}

\author{
Han Bao, Tao Jiang, Kaibin Chu, Mo Chen, Quan Xu, and Bocheng Bao \\ School of Information Science and Engineering, Changzhou University, Changzhou 213164, China \\ Correspondence should be addressed to Bocheng Bao; mervinbao@126.com
}

Received 16 December 2017; Accepted 17 January 2018; Published 25 March 2018

Academic Editor: Viet-Thanh Pham

Copyright (C) 2018 Han Bao et al. This is an open access article distributed under the Creative Commons Attribution License, which permits unrestricted use, distribution, and reproduction in any medium, provided the original work is properly cited.

\begin{abstract}
This paper investigates extreme multistability and its controllability for an ideal voltage-controlled memristor emulator-based canonical Chua's circuit. With the voltage-current model, the initial condition-dependent extreme multistability is explored through analyzing the stability distribution of line equilibrium point and then the coexisting infinitely many attractors are numerically uncovered in such a memristive circuit by the attraction basin and phase portraits. Furthermore, based on the accurate constitutive relation of the memristor emulator, a set of incremental flux-charge describing equations for the memristor-based canonical Chua's circuit are formulated and a dimensionality reduction model is thus established. As a result, the initial condition-dependent dynamics in the voltage-current domain is converted into the system parameter-associated dynamics in the flux-charge domain, which is confirmed by numerical simulations and circuit simulations. Therefore, a controllable strategy for extreme multistability can be expediently implemented, which is greatly significant for seeking chaos-based engineering applications of multistable memristive circuits.
\end{abstract}

\section{Introduction}

Initial condition-dependent extreme multistability, first encountered in several coupled nonlinear dynamical systems [1-3], is a coexisting phenomenon of infinitely many attractors for a given set of system parameters. More recently, due to the existence of infinitely many equilibrium points, for example, line equilibrium point or plane equilibrium point, this special dynamical phenomenon of extreme multistability is naturally exhibited in a class of ideal flux/voltage-controlled memristor-based chaotic circuits/systems [4-9], thereby leading to the emergence of infinitely many disconnected attractors.

Extreme multistability is a fantastic kind of multistability, which makes a nonlinear dynamical circuit or system supply great flexibility for its potential uses in chaos-based engineering applications [10-12], but also raises new challenges for its control of the existing multiple stable states [11-14]. Generally, multistability is confirmed in hardware experiments by randomly switching on and off experimental circuit supplies $[9,15-21]$ or by MATLAB numerical or PSPICE/PSIM circuit simulations [4-8, 22-28]. Consequently, to direct the nonlinear dynamical circuit or system to a desired oscillating mode, an effective control approach should be proposed [12]. To this end, this paper takes an ideal voltage-controlled memristor emulator-based canonical Chua's circuit as an example; a controllable strategy for extreme multistability is achieved through converting the initial condition-dependent dynamics in the voltage-current domain into the system parameter-associated dynamics in the flux-charge domain $[29,30]$.

Besides, for a memristor-based circuit or system with line equilibrium point or plane equilibrium point, its stability at the equilibrium point is very difficult to be determined due to the existence of one or two zero eigenvalues [5-9], which results in the fact that the coexisting infinitely many attractors' behaviors can not be precisely interpreted from the stabilities of the nonzero eigenvalues. As a matter of fact, 


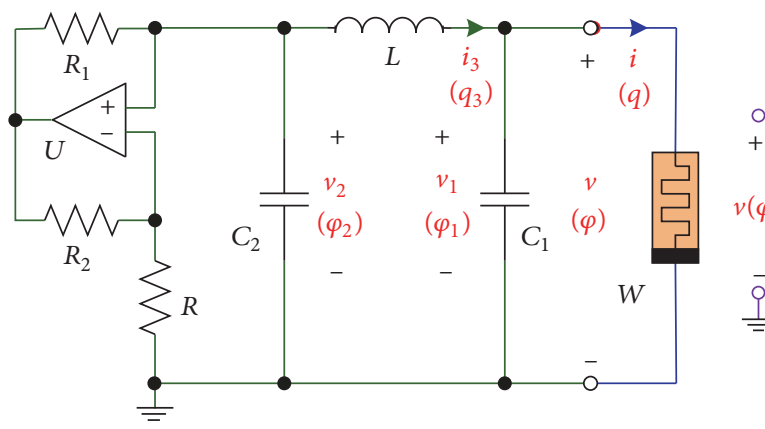

(a)

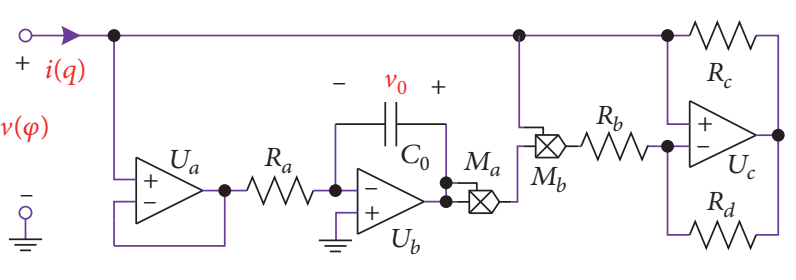

(b)

Figure 1: Memristor-based canonical Chua's circuit. (a) Circuit schematic with simple topology. (b) Ideal voltage-controlled memristor emulator implemented with discrete components.

the memristor initial condition and other initial conditions all have dynamical effects on the memristor-based circuit or system $[8,9]$. However, the dynamical effects are implied, which can not be explicitly expressed in the voltage-current domain. How about the memristor-based circuit or system in the flux-charge domain?

Flux-charge analysis method was first postulated as a tool of dimensionality reduction [31-36], in which the initial conditions of the memristor-based circuit or system are not precisely formulated, thereby leading to the absence of the initial condition-dependent dynamical behaviors [34-36]. In the last two years, a new flux-charge analysis method is reported in $[29,30]$, which judiciously utilizes the incremental flux and charge to substitute the conventional flux and charge and efficaciously solves the issue of the original fluxcharge analysis method. Accordingly, based on the voltagecurrent relation, an accurate flux-charge relation of the ideal voltage-controlled memristor emulator is established. With the accurate constitutive relation, an incremental flux-charge model for the memristor-based canonical Chua's circuit is constructed, upon which all the initial conditions in the voltage-current model can be explicitly formulated by the system parameters in the flux-charge model and the multiple stable states can be consequently controlled by changing the initial condition-related system parameters.

The rest of the paper is structured as follows. In Section 2, an ideal voltage-controlled memristor emulatorbased canonical Chua's circuit is presented. With the voltagecurrent model, the initial condition-dependent extreme multistability is explored and then the coexisting infinitely many attractors are numerically uncovered. In Section 3, based on the accurate constitutive relation of the memristor emulator, a set of incremental flux-charge equations for the memristor-based canonical Chua's circuit are formulated and a dimensionality reduction model is thus established, upon which the feasibility of the flux-charge analysis method is verified by MATLAB numerical simulations. In Section 4, an equivalent circuit of the incremental flux-charge model is designed and circuit simulations for the initial conditiondependent behaviors are executed, from which the controllability of extreme multistability is physically confirmed. The conclusions are drawn in the last section.
TABLE 1: Circuit parameters of memristor-based canonical Chua's circuit.

\begin{tabular}{lcc}
\hline Parameters & Significations & Values \\
\hline$C_{0}$ & Capacitance & $1 \mathrm{nF}$ \\
$C_{1}$ & Capacitance & $4.7 \mathrm{nF}$ \\
$C_{2}$ & Capacitance & $33 \mathrm{nF}$ \\
$L_{1}$ & Inductor & $30 \mathrm{mH}$ \\
$g$ & Total gain & $0.2 \mathrm{~V}^{-2}$ \\
$R_{a}$ & Resistance & $4 \mathrm{k} \Omega$ \\
$R_{b}$ & Resistance & $1.5 \mathrm{k} \Omega$ \\
$R_{c}, R_{d}$ & Resistance & $2 \mathrm{k} \Omega$ \\
$R$ & Resistance & $1.5 \mathrm{k} \Omega$ \\
$R_{1}, R_{2}$ & Resistance & $2 \mathrm{k} \Omega$ \\
\hline
\end{tabular}

\section{Extreme Multistability in the Voltage-Current Domain}

Based on a canonical Chua's circuit and an ideal voltagecontrolled memristor emulator, a new memristor-based canonical Chua's circuit is constructed, as shown in Figure 1(a), which is simple and physically realizable. The ideal voltage-controlled memristor emulator is equivalently implemented with an electronic circuit via op-amp integrators and analog multipliers [5, 6, 34], as shown in Figure 1(b). In our next work, the considered circuit parameters remained unchanged and are listed in Table 1, where $g$ is the total gain of two multipliers $M_{a}$ and $M_{b}$.

2.1. Conventional Voltage-Current Model. For the ideal voltage-controlled memristor emulator in Figure 1(b), the relationships of the input voltage $v$, the input current $i$, and the voltage $v_{0}$ of the capacitor $C_{0}$ can be mathematically described in the voltage-current domain as

$$
\begin{aligned}
C_{0} \frac{\mathrm{d} v_{0}}{\mathrm{~d} t} & =-\frac{1}{R_{a}} v \\
i & =W\left(v_{0}\right) v=-\frac{1}{R_{b}}\left(1-g v_{0}^{2}\right) v .
\end{aligned}
$$


Thus, for the state variables of $v_{0}, v_{1}, v_{2}$, and $i_{3}$ in Figure 1 , the describing circuit equations are easily given in the voltagecurrent domain as

$$
\begin{aligned}
C_{0} \frac{\mathrm{d} v_{0}}{\mathrm{~d} t} & =-\frac{1}{R_{a}} v_{1}, \\
C_{1} \frac{\mathrm{d} v_{1}}{\mathrm{~d} t} & =\frac{1}{R_{b}}\left(1-g v_{0}^{2}\right) v_{1}+i_{3}, \\
C_{2} \frac{\mathrm{d} v_{2}}{\mathrm{~d} t} & =\frac{v_{2}}{R}-i_{3}, \\
L \frac{\mathrm{d} i_{3}}{\mathrm{~d} t} & =v_{2}-v_{1},
\end{aligned}
$$

where $v=v_{1}$.

Introduce four new state variables and scale the circuit parameters as

$$
\begin{aligned}
x_{1} & =v_{0}, \\
x_{2} & =v_{1}, \\
x_{3} & =v_{2}, \\
x_{4} & =R i_{3}, \\
\tau & =\frac{t}{R C_{2}}, \\
R_{b} & =R, \\
a & =\frac{R C_{2}}{R_{a} C_{0}}, \\
b & =\frac{C_{2}}{C_{1}}, \\
c & =\frac{R^{2} C_{2}}{L} .
\end{aligned}
$$

Model (2) can be reexpressed as

$$
\begin{aligned}
& \dot{x}_{1}=-a x_{2}, \\
& \dot{x}_{2}=b\left(1-g x_{1}^{2}\right) x_{2}+b x_{4}, \\
& \dot{x}_{3}=x_{3}-x_{4}, \\
& \dot{x}_{4}=c\left(x_{3}-x_{2}\right),
\end{aligned}
$$

which indicates that there are only four parameters in the normalized system model.

With the circuit parameters in Table 1 , the normalized parameters for model (4) are obtained by (3) as

$$
\begin{aligned}
& a=12.375, \\
& b=7.0213, \\
& c=2.475, \\
& g=0.2 .
\end{aligned}
$$

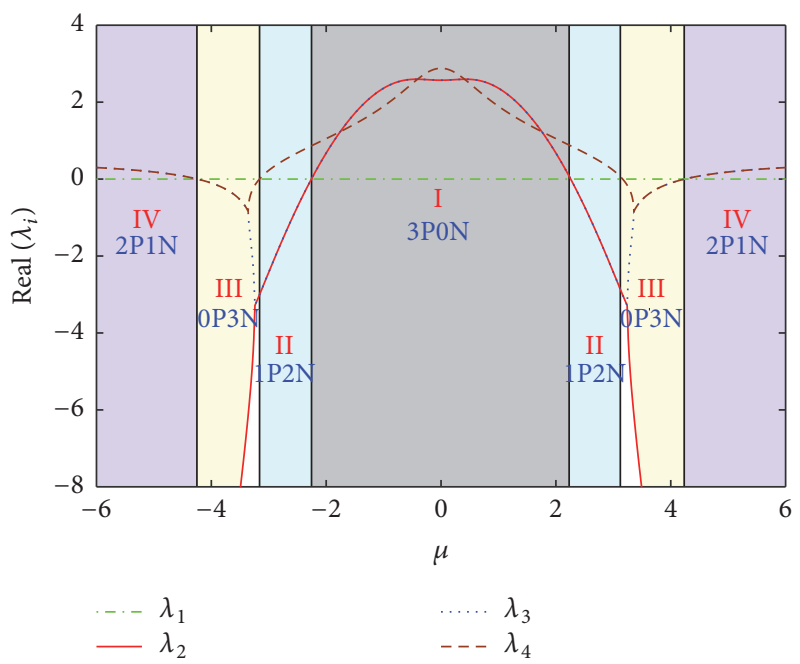

FIGURE 2: Stability distributions classified by the real parts of three nonzero eigenvalues of the line equilibrium point $P$ in the region of $-6 \leq \mu \leq 6$, where $i=1,2,3,4$.

In the following works, the memristor-based canonical Chua's circuit modeled by (4) and the typical system parameters given by (5) are utilized.

2.2. Stability Distribution of Line Equilibrium Point. Similar to the memristive Chua's circuit containing an ideal voltagecontrolled memristor emulator $[5,6]$, the memristor-based canonical Chua's circuit has a line equilibrium point, which is expressed by

$$
P=\left\{\left(x_{1}, x_{2}, x_{3}, x_{4}\right) \mid x_{2}=x_{3}=x_{4}=0, x_{1}=\mu\right\},
$$

where the constant $\mu$ is uncertain.

At the line equilibrium point $P$, the Jacobian matrix is given as

$$
\mathbf{J}_{P}=\left[\begin{array}{cccc}
0 & -a & 0 & 0 \\
0 & b\left(1-g \mu^{2}\right) & 0 & b \\
0 & 0 & 1 & -1 \\
0 & -c & c & 0
\end{array}\right] .
$$

For the Jacobian matrix given in (7), the normalized parameters determined in (5), and the constant $\mu$ that increased in the region $[-6,6]$, four eigenvalues with a zero root, a real root, and a pair of conjugated complex roots can be calculated by MATLAB numerical simulations. The real parts of these four eigenvalues are drawn in Figure 2, which can be used to classify the stability distributions. It can be seen from Figure 2 that the sign of the real parts of three nonzero eigenvalues varies with the increase of $\mu$, leading to the occurrence of three kinds of unstable regions marked with I, II, and IV and a kind of stable region marked with III. Additionally, it can also be observed that the stability distributions of the nonzero eigenvalues in the negative region of $\mu$ are symmetrical to those in the positive region of $\mu$. 
TABLE 2: Nonzero eigenvalues and attractor types for different memristor initial conditions.

\begin{tabular}{|c|c|c|c|}
\hline$x_{1}(0)$ & Nonzero eigenvalues & Stability regions & Attractor types \\
\hline 0 & $\begin{array}{l}2.8804,2.5704 \pm j 2.3364 \\
\quad(\text { Unstable node-foci) }\end{array}$ & Region I: 3P0N & $\begin{array}{l}\text { Double-scroll chaotic } \\
\text { attractor }\end{array}$ \\
\hline 2 & $\begin{array}{c}1.0514,0.6765 \pm j 4.4019 \\
\text { (Unstable node-foci) }\end{array}$ & Region I: 3P0N & Infinite \\
\hline 2.4 & $\begin{array}{c}0.7591,-0.4132 \pm j 4.3865 \\
\text { (Unstable saddle-foci) }\end{array}$ & Region II: 1P2N & Chaotic spiral attractor \\
\hline 3.2 & $\begin{array}{c}-3.1446 \pm j 1.4734,-0.0692 \\
\quad(\text { Stable node-foci) }\end{array}$ & Region III: 0P3N & Stable point attractor \\
\hline 4.3 & $\begin{array}{c}0.0203 \pm j 1.2808,-17.9841 \\
\quad(\text { Unstable saddle-foci) }\end{array}$ & Region VI: 2P1N & Stable point attractor \\
\hline 4.5 & $\begin{array}{c}0.0800 \pm j 1.3134,-20.5750 \\
\text { (Unstable saddle-foci) }\end{array}$ & Region VI: 2P1N & Limit cycle with period 1 \\
\hline 4.8 & $\begin{array}{c}0.1486 \pm j 1.3483,-24.6301 \\
\text { (Unstable saddle-foci) }\end{array}$ & Region VI: 2P1N & $\begin{array}{l}\text { Asymmetric double- } \\
\text { scroll chaotic attractor }\end{array}$ \\
\hline
\end{tabular}

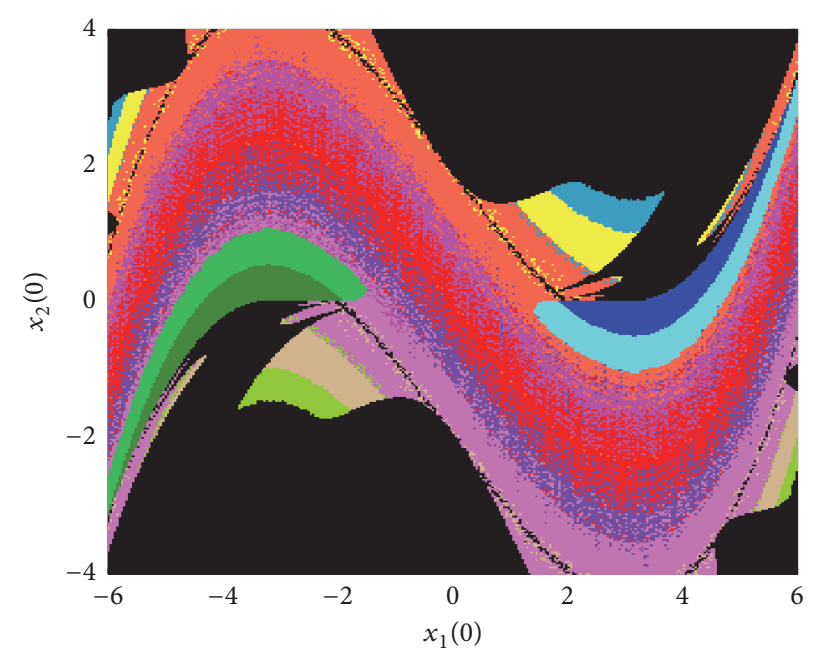

FIGURE 3: Attraction basin in the $x_{1}(0)-x_{2}(0)$ plane, where $a=12.375, b=7.0213, c=2.475, g=0.2$, and $x_{3}(0)=x_{4}(0)=0$.

Due to the existence of the zero eigenvalue, the stability of the memristor-based canonical Chua's circuit can not be simply determined by the three nonzero eigenvalues of the line equilibrium point. The following numerical simulations demonstrate that the zero eigenvalue also has influence on the dynamics of the circuit under some circuit parameters [6-9].

The initial conditions for numerical simulations of the coexisting attractors' behaviors are taken as $\left[x_{1}(0), 10^{-9}\right.$, $0,0]$; that is, only the memristor initial condition $x(0)$ is variable. For some different values of the memristor initial condition $x_{1}(0)$, nonzero eigenvalues, stability regions given in Figure 2, and the related attractor types numerically solved by (4) are summarized in Table 2 . It is demonstrated that, for the different values of $x_{1}(0)$ located in different stability regions, there exist various attractor types with different topologies or different periodicities or different locations. Consequently, coexisting infinitely many attractors' behavior or extreme multistability occurs in the memristor-based canonical Chua's circuit.

2.3. Coexisting Infinitely Many Attractors. With reference to the stability distributions in Figure 2 and the initial condition-dependent attractor types in Table 2, the proposed memristor-based canonical Chua's circuit has various stable states under different initial conditions; that is, its long-term behavior closely relies on the initial conditions therefore leading to the emergence of coexisting infinitely many attractors.

For the normalized parameters in (5) and the initial conditions of $x_{3}(0)=0$ and $x_{4}(0)=0$, the attraction basin in the plane of the initial conditions of $x_{1}(0)$ and $x_{2}(0)$ is depicted in Figure 3. It should be illustrated that many more diverse attractor types for different initial conditions can be certainly observed in the memristor-based canonical Chua's circuit; however, for visual effects, only fourteen kinds 
TABLE 3: Different color regions and the corresponding attractor types.

\begin{tabular}{lcc}
\hline Colors & Coexisting attractor types & Examples in Figure 4 \\
\hline Blue and forest green & Right- and left-point attractors & Figure 4(a) \\
Cyan and lime green & Right- and left-period-1 limit cycles with small size & Figure 4(b) \\
Cadet blue and lawn green & Left- and right-period-1 limit cycles with large size & Figure 4(c) \\
Tan and yellow & Left- and right-multi-period limit cycles & Figure 4(d) \\
Orchid and coral & Left- and right-chaotic spiral attractors & Figure 4(e) \\
Medium slate blue and fuchsia & Left- and right-half-baked double-scroll chaotic attractors & Figure 4(f) \\
Red & Standard double-scroll chaotic attractor & Figure 4(g) \\
Black & Unbounded orbit & Figure 4(h) \\
\hline
\end{tabular}

of color areas are classified by the basin of attraction in the $x_{1}(0)-x_{2}(0)$ plane, among which the largest black area represents the unbounded zone.

Corresponding to different color areas, different types of coexisting attractors are listed in Table 3. Spontaneously, for the initial conditions in the different color areas of the attraction basin, the phase portraits of typical coexisting attractors are obtained, as shown in Figure 4, where for the sake of observations two point attractors in Figure 4(a) are marked by two five-pointed stars. Of course, these generated coexisting attractors intersect the neighborhood of the line equilibrium point, implying that the initials-dependent dynamical system (4) [37] always oscillates in self-excited states, rather than hidden states [38-40].

It should be mentioned that just like the ideal flux/ voltage-controlled memristor-based chaotic circuits [4-9], the proposed memristor-based canonical Chua's circuit has a line equilibrium point with complicated stability distributions already depicted in Figures 2-4, whereas most of conventionally nonlinear dynamical systems with no equilibrium point [10], with only several determined equilibrium points [15-21], or with curves of equilibrium points [41-43] have relatively simple stability distributions with some divinable nonlinear dynamical behaviors.

\section{Controllability of Extreme Multistability in the Flux-Charge Domain}

Due to the existence of the line equilibrium point, the memristor-based canonical Chua's circuit can exhibit the special phenomenon of extreme multistability under different initial conditions. For seeking the potential uses of the multistable memristive circuit in chaos-based engineering applications [10-12], an effective control method should be applied to direct the memristive circuit to the desired oscillation mode [12]. For this purpose, an incremental flux-charge model is newly constructed, in which the initial condition-dependent dynamics in the voltage-current domain is converted into the system parameter-associated dynamics in the flux-charge domain.

3.1. Newly Constructed Incremental Flux-Charge Model. The accurate constitutive relation of the ideal voltagecontrolled memristor emulator in Figure 1(b) should be firstly established in the flux-charge domain. Define $q(t ; 0)$ and $\varphi(t ; 0)$ as the incremental charge and incremental flux of the ideal memristor emulator, respectively. According to (1), the incremental charge within the time interval $[0, t]$ is deduced as

$$
\begin{aligned}
q(t ; 0)= & \int_{0}^{t} i \mathrm{~d} \tau=\int_{0}^{t}-\frac{1}{R_{b}}\left(1-g v_{0}^{2}\right) v_{1} \mathrm{~d} \xi \\
= & \frac{1}{R_{b}} \int_{0}^{t}\left(1-g v_{0}^{2}\right) R_{a} C_{0} \mathrm{~d} v_{0} \\
= & \frac{1}{\kappa R_{b}}\left[v_{0}(t)-v_{0}(0)\right]-\frac{g}{3 \kappa R_{b}}\left[v_{0}^{3}(t)-v_{0}^{3}(0)\right] \\
= & \frac{g \kappa^{2}}{3 R_{b}}[\varphi(t ; 0)]^{3}-\frac{g \kappa}{R_{b}} v_{0}(0)[\varphi(t ; 0)]^{2} \\
& +\frac{1}{R_{b}}\left[g v_{0}^{2}(0)-1\right] \varphi(t ; 0)
\end{aligned}
$$

where $\kappa=1 /\left(R_{a} C_{0}\right), v_{0}(0)$ stands for the memristor initial state, and the memristor inner state variable $v_{0}(t)=$ $-\kappa \varphi(t ; 0)+v_{0}(0)$. Thus, the initial state $v_{0}(0)$ can be explicitly represented in the flux-charge domain.

Suppose that $\varphi_{1}(t ; 0), \varphi_{2}(t ; 0)$, and $q_{3}(t ; 0)$ are the incremental fluxes of the capacitors $C_{1}$ and $C_{2}$ and the incremental charge of the inductor $L$, respectively, and set $v_{1}(0), v_{2}(0)$, and $i_{3}(0)$ as the initial voltages of the capacitors $C_{1}$ and $C_{2}$ and the initial current of the inductor $L$, respectively. While connecting the power supply at $t=0$, the incremental flux-charge model of the memristor-based canonical Chua's circuit can be yielded by integrating (2) from 0 to $t$.

Integrating the second, third, and fourth equations of (2) from 0 to $t$ gives

$$
\begin{aligned}
C_{1}\left[v_{1}(t)-v_{1}(0)\right] & =-q(t ; 0)+q_{3}(t ; 0), \\
C_{2}\left[v_{2}(t)-v_{2}(0)\right] & =\frac{1}{R} \varphi_{2}(t ; 0)-q_{3}(t ; 0), \\
L\left[i_{3}(t)-i_{3}(0)\right] & =\varphi_{2}(t ; 0)-\varphi_{1}(t ; 0) .
\end{aligned}
$$

Consider the fact that the fluxes $\varphi_{1}(t)$ and $\varphi_{2}(t)$ of the capacitors $C_{1}$ and $C_{2}$ and the charge $q_{3}(t)$ of the inductor $L$ can be expressed as

$$
\varphi_{1}(t)=\int_{-\infty}^{t} v_{1}(\xi) \mathrm{d} \xi=\varphi_{1}(t ; 0)+K_{1}
$$




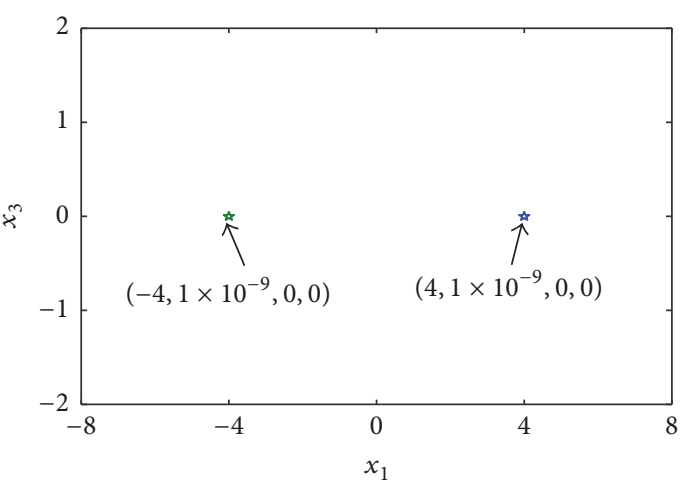

(a)

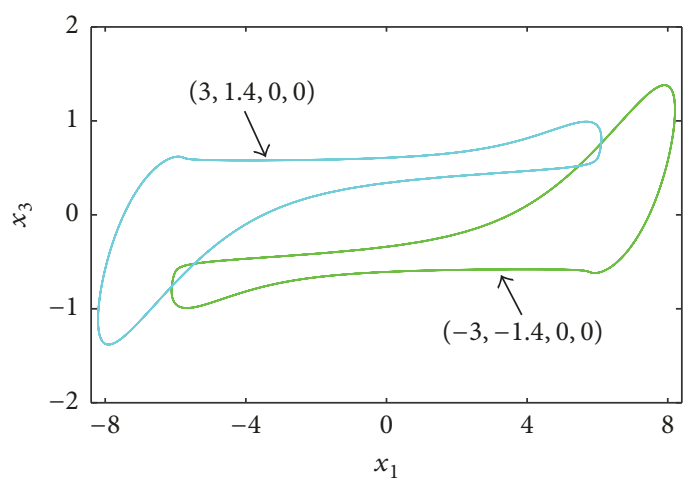

(c)

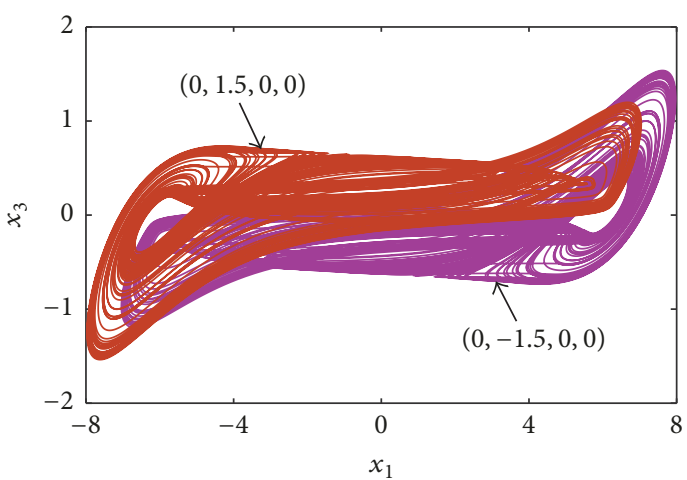

(e)

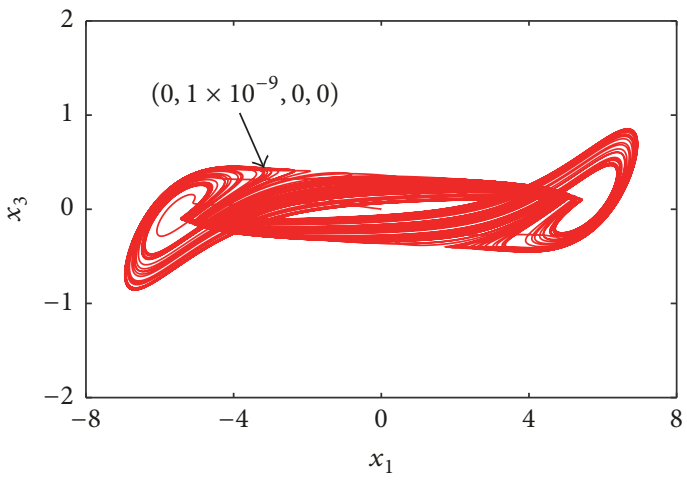

(g)

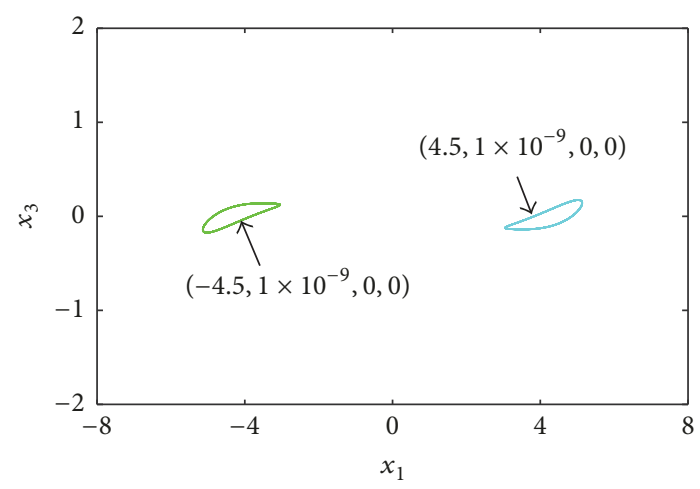

(b)

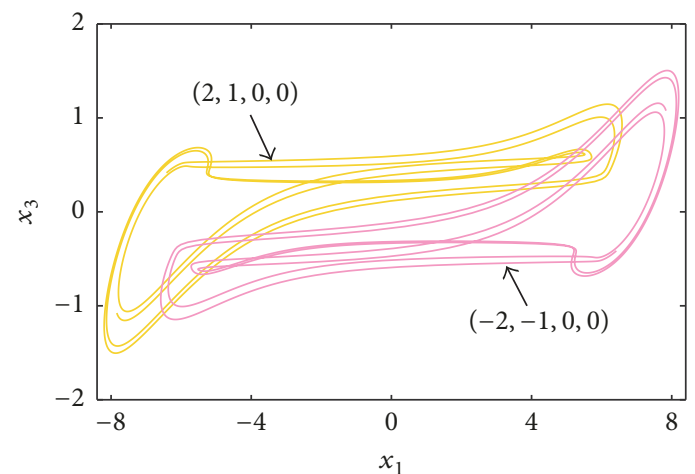

(d)

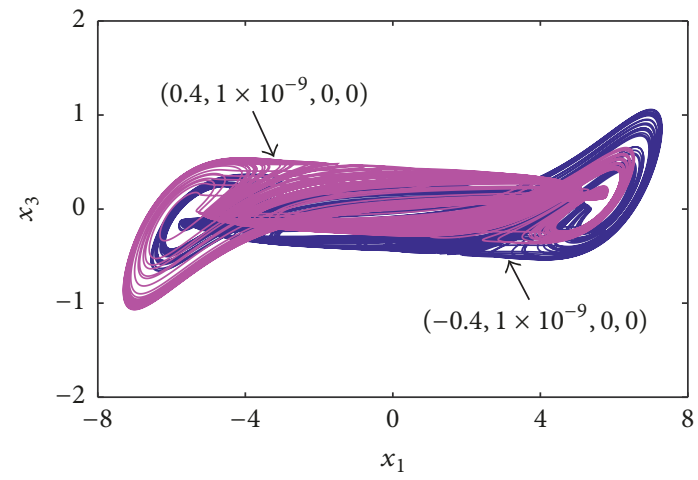

(f)

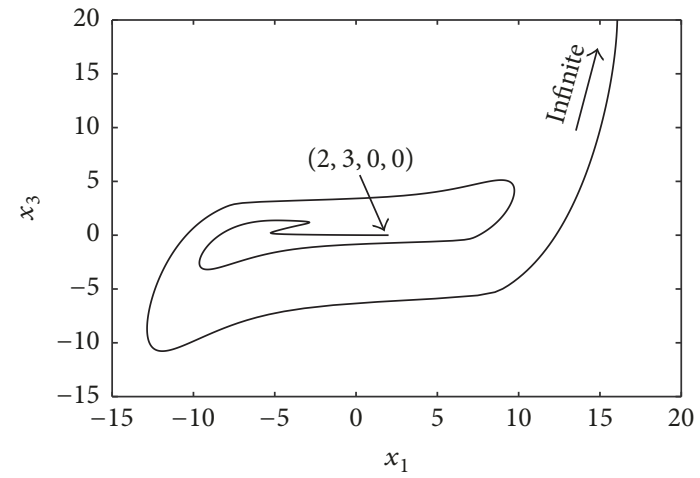

(h)

FIGURE 4: Phase portraits of coexisting infinitely many attractors in the $x_{1}-x_{3}$ plane for different values of $x_{1}(0)$ and $x_{2}(0)$. (a) Right- and left-point attractors. (b) Right- and left-period-1 limit cycles with small size. (c) Left- and right-period-1 limit cycles with large size. (d) Leftand right-multiperiod limit cycles. (e) Left- and right-chaotic spiral attractors. (f) Left- and right-half-baked double-scroll chaotic attractors. (g) Standard double-scroll chaotic attractor. (h) Unbounded orbit. 


$$
\begin{aligned}
& \varphi_{2}(t)=\int_{-\infty}^{t} v_{2}(\xi) \mathrm{d} \xi=\varphi_{2}(t ; 0)+K_{2}, \\
& q_{3}(t)=\int_{-\infty}^{t} i_{3}(\xi) \mathrm{d} \xi=q_{3}(t ; 0)+K_{3},
\end{aligned}
$$

respectively, where $K_{1}=\int_{-\infty}^{0} v_{1}(\xi) \mathrm{d} \xi, K_{2}=\int_{-\infty}^{0} v_{2}(\xi) \mathrm{d} \xi$, and $K_{3}=\int_{-\infty}^{0} i_{3}(\xi) \mathrm{d} \xi$ represent three arbitrary real constants. Therefore, the state variables $v_{1}(t), v_{2}(t)$, and $i_{3}(t)$ in the voltage-current domain can be signified as

$$
\begin{aligned}
& v_{1}(t)=\frac{\mathrm{d} \varphi_{1}(t)}{\mathrm{d} t}=\frac{\mathrm{d} \varphi_{1}(t ; 0)}{\mathrm{d} t}, \\
& v_{2}(t)=\frac{\mathrm{d} \varphi_{2}(t)}{\mathrm{d} t}=\frac{\mathrm{d} \varphi_{2}(t ; 0)}{\mathrm{d} t}, \\
& i_{3}(t)=\frac{\mathrm{d} q_{3}(t)}{\mathrm{d} t}=\frac{\mathrm{d} q_{3}(t ; 0)}{\mathrm{d} t} .
\end{aligned}
$$

Substituting (11) into (9), the circuit equations of Figure 1 can be thereby modeled in the flux-charge domain as

$$
\begin{aligned}
C_{1} \frac{\mathrm{d} \varphi_{1}(t ; 0)}{\mathrm{d} t} & =-q(t ; 0)+q_{3}(t ; 0)+C_{1} v_{1}(0), \\
C_{2} \frac{\mathrm{d} \varphi_{2}(t ; 0)}{\mathrm{d} t} & =\frac{1}{R} \varphi_{2}(t ; 0)-q_{3}(t ; 0)+C_{2} v_{2}(0), \\
L \frac{\mathrm{d} q_{3}(t ; 0)}{\mathrm{d} t} & =\varphi_{2}(t ; 0)-\varphi_{1}(t ; 0)+L i_{3}(0) .
\end{aligned}
$$

Equation (12) is the incremental flux-charge model of the proposed memristor-based canonical Chua's circuit.

Analogously, introduce three new state variables and scale the circuit parameters as

$$
\begin{aligned}
y_{1} & =\frac{\varphi_{1}(t ; 0)}{R C_{2}}, \\
y_{2} & =\frac{\varphi_{2}(t ; 0)}{R C_{2}}, \\
y_{3} & =\frac{q_{3}(t ; 0)}{C_{2}}, \\
\tau & =\frac{t}{R C_{2}}, \\
R_{b} & =R, \\
a & =\frac{R C_{2}}{R_{a} C_{0}}, \\
b & =\frac{C_{2}}{C_{1}}, \\
c & =\frac{R^{2} C_{2}}{L}, \\
\eta & =v_{0}(0), \\
\eta_{1} & =v_{1}(0),
\end{aligned}
$$

$$
\begin{aligned}
& \eta_{2}=v_{2}(0), \\
& \eta_{3}=R i_{3}(0) .
\end{aligned}
$$

Model (12) can be rewritten as

$$
\begin{aligned}
& \dot{y}_{1}=-b F\left(y_{1}\right)+b y_{3}+\eta_{1}, \\
& \dot{y}_{2}=y_{2}-y_{3}+\eta_{2}, \\
& \dot{y}_{3}=c\left(y_{2}-y_{1}\right)+\eta_{3},
\end{aligned}
$$

where the normalized memristor constitutive relation is turned as $F\left(y_{1}\right)=(1 / 3) g a^{2} y_{1}^{3}-g a \eta y_{1}^{2}+\left(g \eta^{2}-1\right) y_{1}$.

It should be emphasized that the initial conditions of (14) are ensured as $y_{1}(0)=y_{2}(0)=y_{3}(0)=0$. The memristor inner parameter $\eta$ denotes the initial capacitor voltage of the memristor emulator in the voltage-current domain, and the system parameters $\eta_{1}, \eta_{2}$, and $\eta_{3}$ reflect the three initial voltages of the canonical Chua's circuit in the voltage-current domain.

For the circuit parameters given in Table 1, the normalized parameters $a, b, c$, and $g$ in (14) are the same as those given in (5). With these determined parameters, the initial conditions-dependent extreme multistability in the memristor-based canonical Chua's circuit can be effectively controlled by adjusting the system parameters $\eta, \eta_{1}, \eta_{2}$, and $\eta_{3}$.

3.2. System Parameter-Related Stability Distribution. For the normalized model (14), the equilibrium points are obviously obtained as

$$
E=\left(\bar{y}_{1}, \bar{y}_{1}-\frac{1}{c} \eta_{3}, \bar{y}_{1}+\eta_{2}-\frac{1}{c} \eta_{3}\right),
$$

in which $\bar{y}_{1}$ can be numerically solved by

$$
\begin{aligned}
\bar{y}_{1}^{3}-\frac{3 \eta}{a} \bar{y}_{1}^{2}+\frac{3}{g a^{2}}\left(g \eta^{2}-2\right) \bar{y}_{1} \\
-\frac{3 c \eta_{1}+3 b c \eta_{2}-3 b \eta_{3}}{g a^{2} b c}=0 .
\end{aligned}
$$

Define $P$ and $Q$ to be

$$
\begin{aligned}
& P=\frac{3}{g a^{2}}\left(g \eta^{2}-2\right)-\frac{3 \eta^{2}}{a^{2}}=-\frac{6}{g a^{2}}, \\
& Q=\frac{\eta^{3}}{a^{3}}-\frac{6 \eta}{g a^{3}}-\frac{3 c \eta_{1}+3 b c \eta_{2}-3 b \eta_{3}}{g a^{2} b c} .
\end{aligned}
$$

According to the classical Cardan discriminant $\Delta=(Q / 2)^{2}+$ $(P / 3)^{3}$, when $\Delta<0$, there are three real roots in (16), which can be given as

$$
\bar{y}_{1,1}=\sqrt[3]{-\frac{Q}{2}+\sqrt{\Delta}}+\sqrt[3]{-\frac{Q}{2}-\sqrt{\Delta}}+\frac{\eta}{a},
$$




$$
\begin{aligned}
\bar{y}_{1,2}= & -\frac{1-j \sqrt{3}}{2} \sqrt[3]{-\frac{Q}{2}+\sqrt{\Delta}}-\frac{1+j \sqrt{3}}{2} \sqrt[3]{-\frac{Q}{2}-\sqrt{\Delta}} \\
& +\frac{\eta}{a} \\
\bar{y}_{1,3}= & -\frac{1+j \sqrt{3}}{2} \sqrt[3]{-\frac{Q}{2}+\sqrt{\Delta}}-\frac{1-j \sqrt{3}}{2} \sqrt[3]{-\frac{Q}{2}-\sqrt{\Delta}} \\
& +\frac{\eta}{a},
\end{aligned}
$$

indicating that the model (14) has three equilibrium points.

The Jacobian matrix at equilibrium point $E$ is deduced as

$$
\mathbf{J}_{E}=\left[\begin{array}{ccc}
-b F^{\prime}\left(\bar{y}_{1}\right) & 0 & b \\
0 & 1 & -1 \\
-c & c & 0
\end{array}\right],
$$

where $F^{\prime}\left(\bar{y}_{1}\right)=g a^{2} \bar{y}_{1}^{2}-2 g a \eta \bar{y}_{1}+g \eta^{2}-1$. Consequently, three eigenvalues of the model (14) at $E$ are yielded by solving the following characteristic polynomial:

$$
P(\lambda)=\operatorname{det}(\mathbf{1} \lambda-\mathbf{J})=\lambda^{3}+p_{1} \lambda^{2}+p_{2} \lambda+p_{3}=0,
$$

in which $p_{1}=b F^{\prime}\left(\bar{y}_{1}\right)-1, p_{2}=-b F^{\prime}\left(\bar{y}_{1}\right)+b c+c$, and $p_{3}=$ $b c F^{\prime}\left(\bar{y}_{1}\right)-b c$.

Based on (15)-(20), it can be known that the line equilibrium point described by (6) in the voltage-current domain is converted into several determined equilibrium points represented by (15) in the flux-charge domain, whose locations and stabilities are decided by the initial conditionrelated system parameters $\eta, \eta_{1}, \eta_{2}$, and $\eta_{3}$. Therefore, the extreme multistability in the voltage-current domain can readily be controlled by the system parameters in the fluxcharge domain.

Take $\eta_{1}=\eta_{2}=\eta_{3}=0$ as an example. When the normalized system parameters $a, b, c$, and $g$ for the model (14) are fixed as given in (5) and the relationship of $-2 \sqrt{10} \leq \eta \leq$ $2 \sqrt{10}$ is satisfied, three equilibrium points consisting of one zero equilibrium point and two nonzero equilibrium points are solved from (16) as

$$
\begin{aligned}
& E_{0}=(0,0,0) \\
& E_{ \pm}=\left(\frac{12 \eta \pm \rho}{99}, \frac{12 \eta \pm \rho}{99}, \frac{12 \eta \pm \rho}{99}\right),
\end{aligned}
$$

where $\rho=4 \sqrt{120-3 \eta^{2}}$, which means that the nonzero equilibrium points depended on the initial condition $\eta$ of the memristor emulator.

For the zero equilibrium point $E_{0}$, there exist $F^{\prime}\left(\bar{y}_{1}\right)=$ $g \eta^{2}-1$. In this way, the Jacobian matrix of (19) with the increase of $\eta$ is identical with the Jacobian submatrix obtained from (7) by deleting row 1 and column 1 with the increase of $\mu$. As a consequence, the Jacobian matrix of (19) has three eigenvalues; their stability distributions are the same as those of the nonzero eigenvalues of the Jacobian matrix of (7). Whereas for two nonzero equilibrium points $E_{ \pm}$, the complicated stability distributions can be numerically found as the memristor initial condition $\eta$ is tuned.

3.3. Controllability of Extreme Multistability. With model (14), it is demonstrated that the initial condition-dependent extreme multistability in the memristor-based canonical Chua's circuit is transformed into the system parameterassociated dynamics, therefore leading to the controllability of extreme multistability through directly adjusting the system parameters.

The normalized system parameters $a, b, c$, and $g$ are given in (5) and the initial conditions for (14) are ensured as $y_{1}(0)=y_{2}(0)=y_{3}(0)=0$. Referring to the initial conditions in Figure $4, \eta_{2}=0$ and $\eta_{3}=0$ in (14) remained unchanged, whereas $\eta$ and $\eta_{1}$ are assigned as some different values in the regions $[-6,6]$ and $[-4,4]$, respectively. For different locations of the parameter space constructed by $\eta$ and $\eta_{1}$, various types of disconnected attractors are numerically simulated, as shown in Figure 5, where for the sake of observations two point attractors overlapped together in Figure 5(a) are marked by two five-pointed stars and two limit cycles in Figure 5(b) are marked with bold lines. Obviously, the dynamical behaviors featured by Figure 5 are consistent with those featured by Figure 4, ignoring the computational errors in MATLAB simulation [44], which verify the feasibility of the incremental flux-charge model of the memristor-based canonical Chua's circuit.

\section{Controlling Multiple Stable States in Physical Circuit}

With model (14), the equivalent circuit using analog multipliers and op-amps linked with resistors and/or capacitors $[7,8,45]$ can be handily designed, as shown in Figure 6, which is composed of the linear calculating circuit with three integrating channels and the memristor constitutive relation circuit. In Figure $6, v_{1}, v_{2}$, and $v_{3}$ represent three state variables of the capacitor voltages, respectively, and $R C$ stands for the time constant of the integrators.

According to the fundamental theory of circuit, the circuit state equations of Figure 6 are expressed as

$$
\begin{aligned}
& R C \frac{\mathrm{d} v_{1}}{\mathrm{~d} t}=-\frac{R F\left(v_{1}\right)}{R_{b}}+\frac{R v_{3}}{R_{b}}+\eta_{1}, \\
& R C \frac{\mathrm{d} v_{2}}{\mathrm{~d} t}=v_{2}-v_{3}+\eta_{2}, \\
& R C \frac{\mathrm{d} v_{3}}{\mathrm{~d} t}=\frac{R\left(v_{2}-v_{1}\right)}{R_{c}}+\eta_{3},
\end{aligned}
$$

where $F\left(y_{1}\right)=R v_{1}^{3} / R_{1}-R \eta v_{1}^{2} / R_{2}+R \eta^{2} v_{1} / R_{3}-v_{1}$. Thus, the circuit parameters can be chosen as $R_{b}=R / b, R_{c}=$ $R / c, R_{1}=3 R /\left(g a^{2}\right), R_{2}=R /(g a)$, and $R_{3}=R / g$.

The op-amps OP07CP and multipliers AD633JNZ with $\pm 15 \mathrm{~V}$ power supplies are utilized. The integrating time constant is selected as $R C=10 \mathrm{k} \Omega \times 10 \mathrm{nF}=100 \mu \mathrm{s}$. Thus, for the system parameters given in (5), the circuit parameters in 


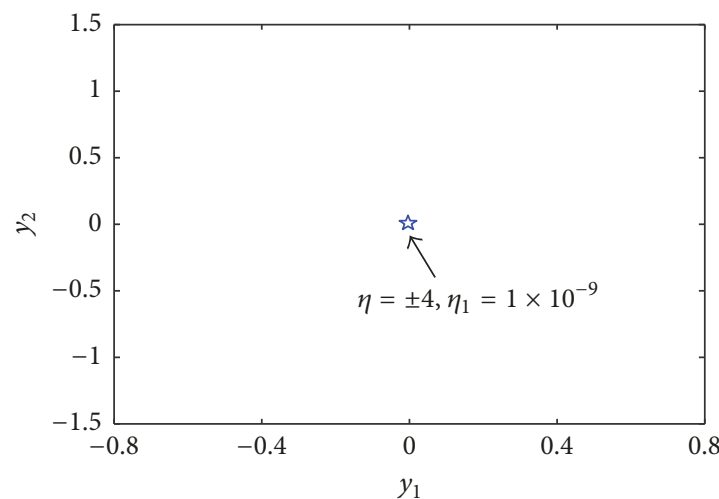

(a)

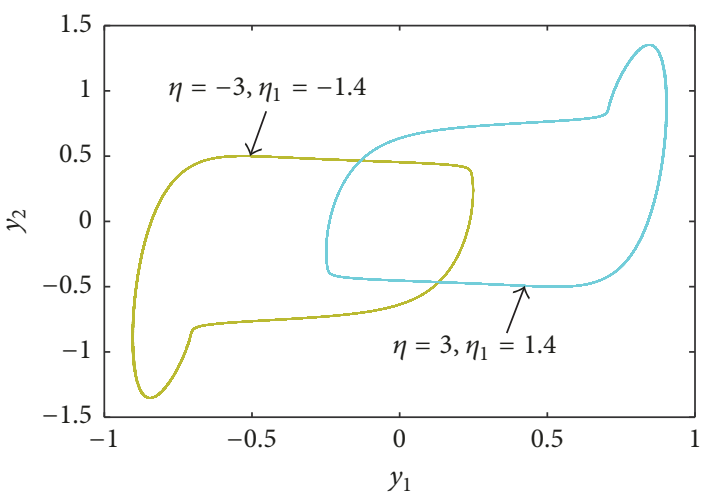

(c)

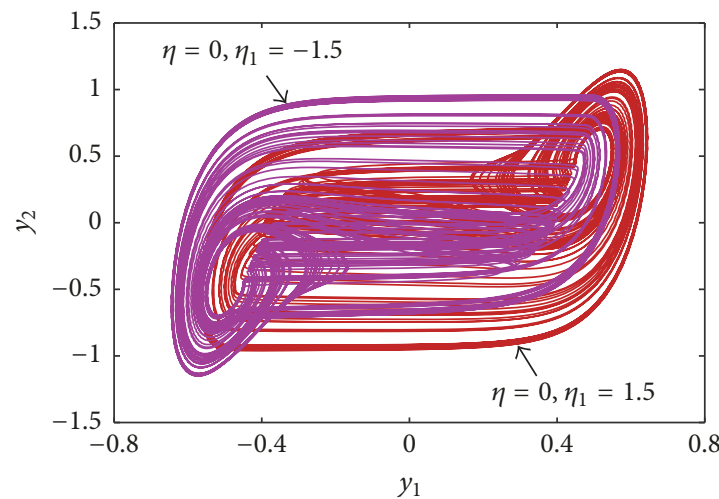

(e)

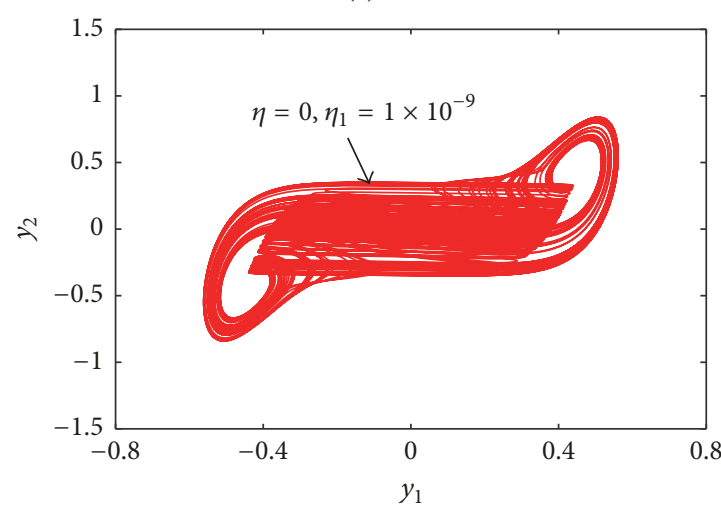

(g)

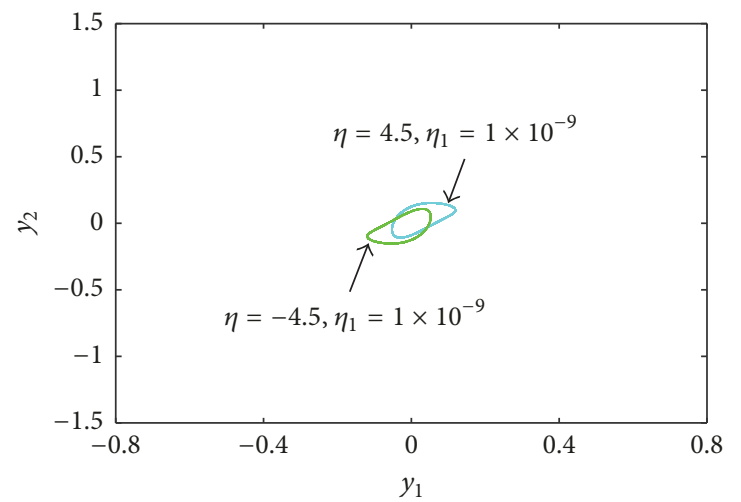

(b)

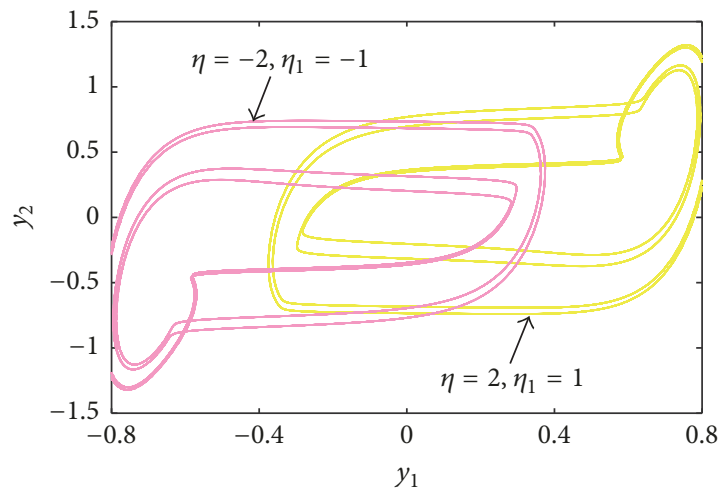

(d)

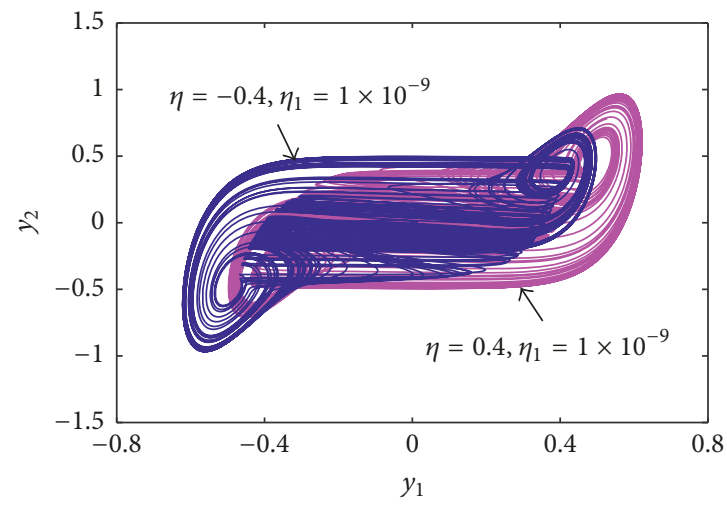

(f)

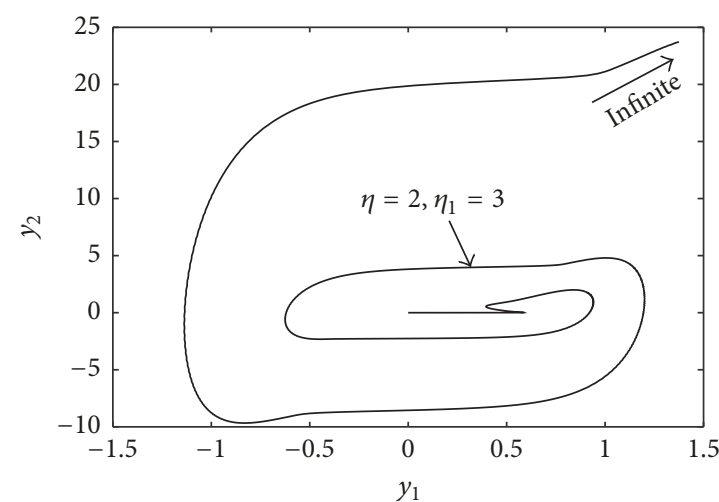

(h)

FIGURE 5: Phase portraits of various types of attractors distributed in different locations of the parameter space of $\eta$ and $\eta_{1}$. (a) Two point attractors. (b) Right- and left-period-1 limit cycles with small size. (c) Right- and left-period-1 limit cycles with large size. (d) Right- and left-multiperiod limit cycles. (e) Right- and left-chaotic spiral attractors. (f) Right- and left-half-baked double-scroll chaotic attractors. (g) Standard double-scroll chaotic attractor. (h) Unbounded orbit. 


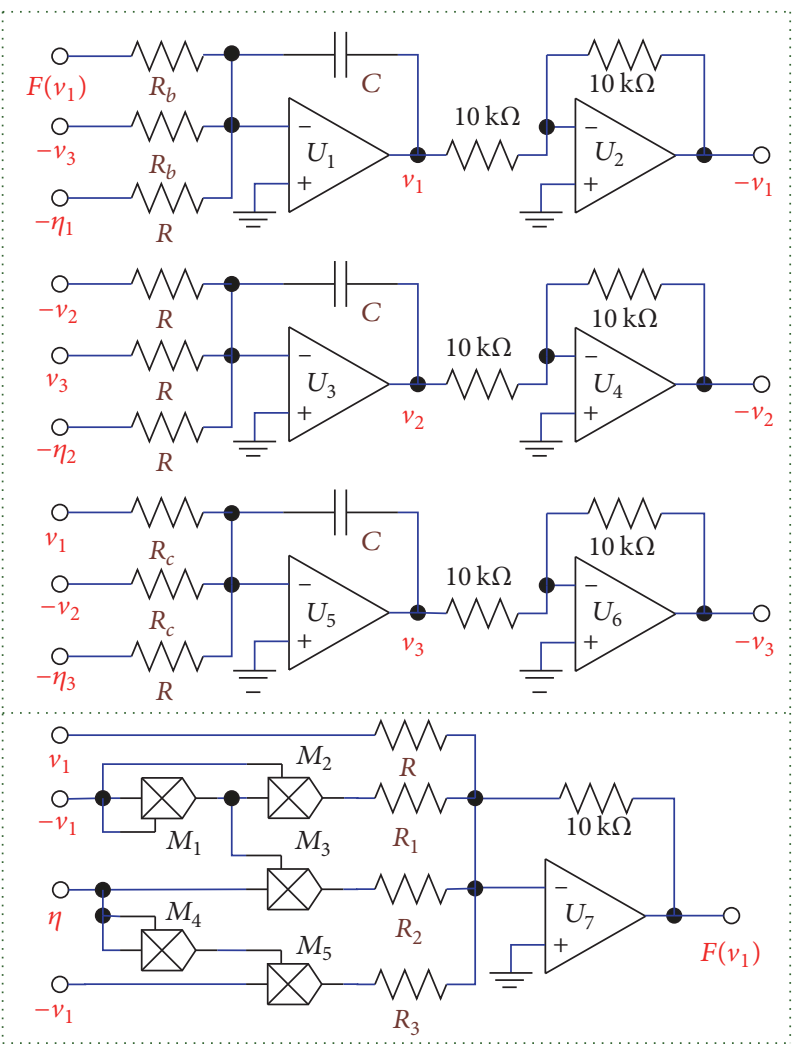

Figure 6: Equivalent circuit of model (14) for controlling multistable states; the upper dashed box is the linear calculating circuit and the lower dashed box is the memristor constitutive relation circuit.

Figure 6 are calculated as $R_{b}=1.4242 \mathrm{k} \Omega, R_{c}=4.0404 \mathrm{k} \Omega, R_{1}$ $=0.9795 \mathrm{k} \Omega, R_{2}=4.0404 \mathrm{k} \Omega$, and $R_{3}=50 \mathrm{k} \Omega$. Additionally, the gains of the multipliers in Figure 6 are all fixed as 1 and the values of $\eta_{2}$ and $\eta_{3}$ are maintained as 0 .

To better present the control effect of the multistable states generated from the equivalent circuit in Figure 6, the NI Multisim 12.0 simulation and circuit design software is utilized, in which the default initial values of three capacitors are assigned as 0 . For several different values of $\eta$ and $\eta_{1}$, the Multisim intercepted phase portraits are displayed, as shown in Figure 7 . Note that the initial value of $1 \times 10^{-9} \mathrm{~V}$ can be achieved by a slightly induced voltage in the equivalent circuit, so the value of $\eta_{1}$ is set as 0 . Comparing the results of Figure 7 with those of Figure 5, it is concluded that the circuit simulations agree with the numerical simulations, further confirming the feasibility of the controllable strategy for extreme multistability.

\section{Conclusion}

By replacing Chua's diode in the canonical Chua's circuit with an ideal voltage-controlled memristor emulator, a memristorbased canonical Chua's circuit is presented in this paper. Because of the existence of a line equilibrium point, the initial condition-dependent extreme multistability easily emerged in such a memristive circuit, resulting in the coexistence of infinitely many attractors. To implement the controllability of the extreme multistability, an incremental flux-charge model for the memristive circuit is formulated through deriving the accurate constitutive relation of the memristor emulator. Thus, the initial condition-dependent dynamics in the voltage-current domain is converted into the system parameter-associated dynamics in the flux-charge domain, that is, the implicit expression of the initial conditions in the voltage-current model can be transformed into the explicit representation of the system parameters in the flux-charge model, leading to the fact that the multiple steady states emerging in the memristive circuit can be consequently controlled by changing the initial condition-related system parameters. The feasibility of the controllable strategy for extreme multistability is confirmed by numerical simulations and circuit simulations, which is greatly significant for seeking the potential uses of the multistable memristive circuits in chaos-based engineering applications.

\section{Conflicts of Interest}

The authors declare that they have no conflicts of interest.

\section{Acknowledgments}

This work was supported by the National Natural Science Foundations of China under Grants nos. 51777016, 61601062, 


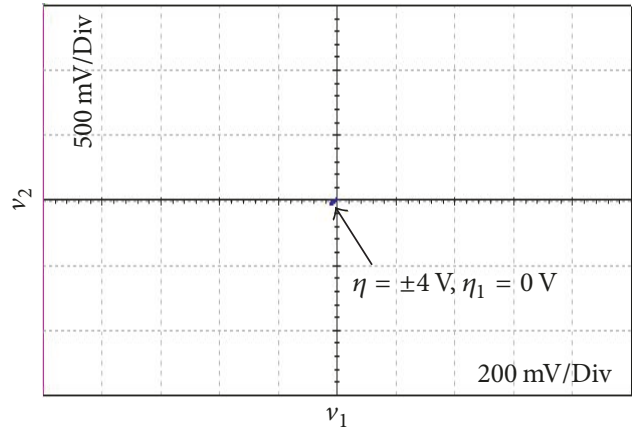

(a)

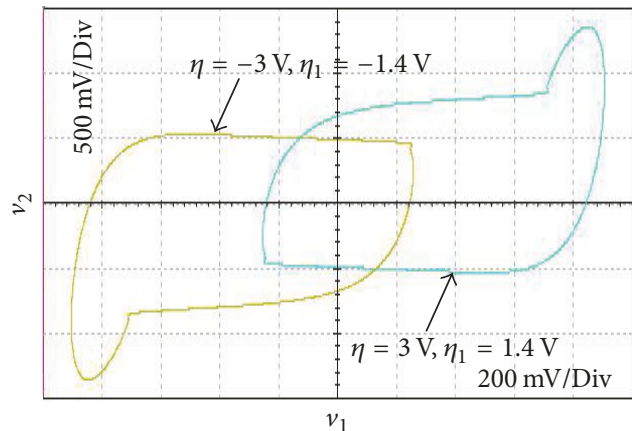

(c)

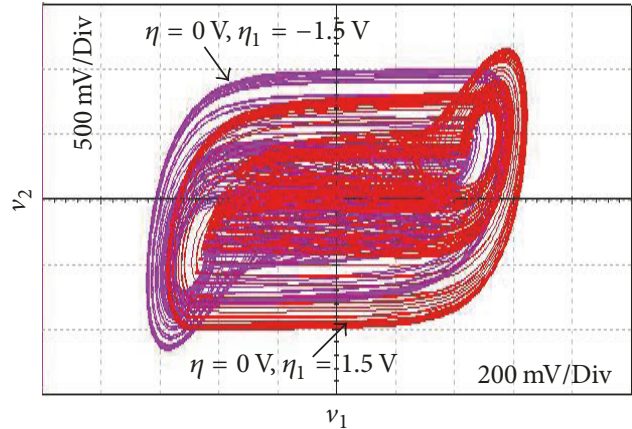

(e)

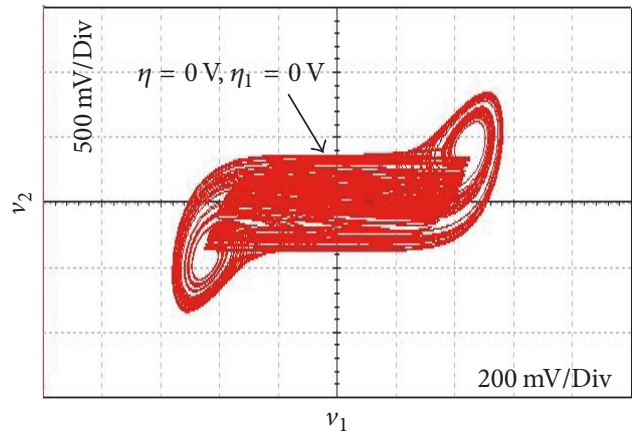

(g)

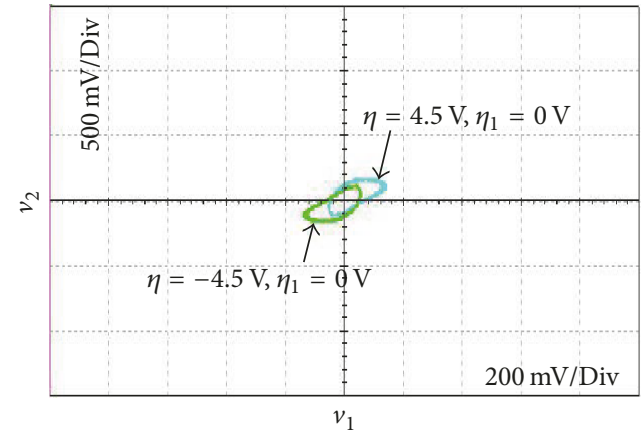

(b)

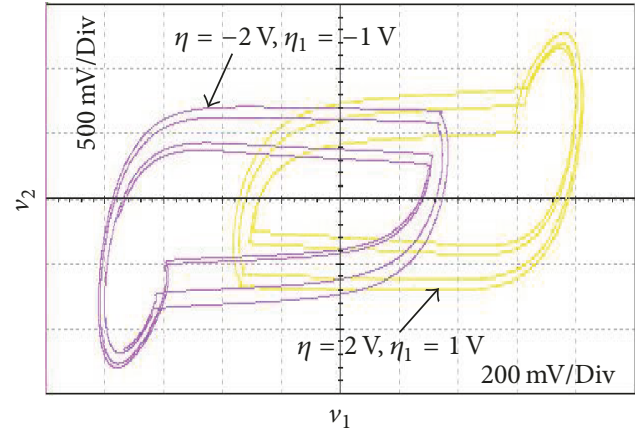

(d)

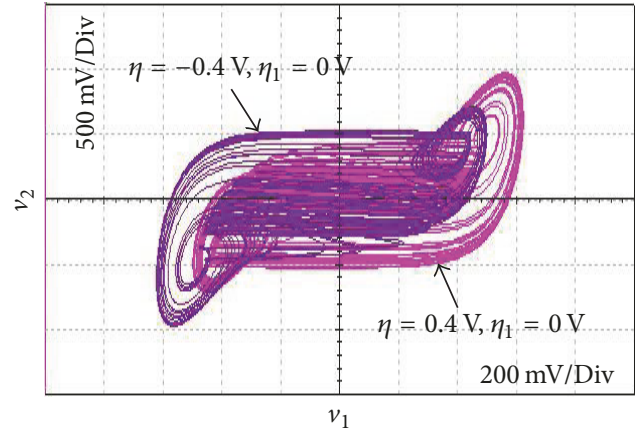

(f)

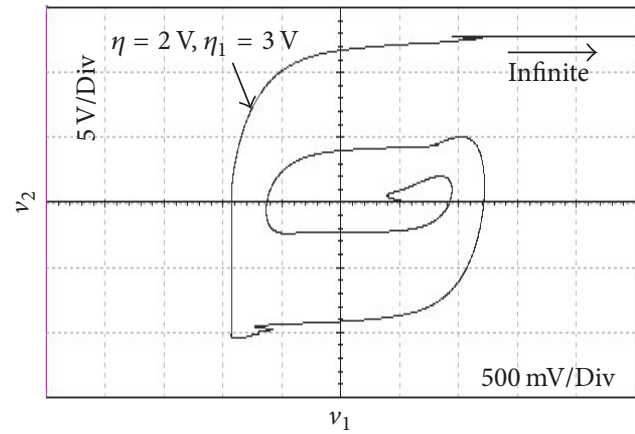

(h)

Figure 7: Multisim intercepted phase portraits of various types of attractors for different values of $\eta$ and $\eta_{1}$. (a) Two point attractors. (b) Right- and left-period-1 limit cycles with small size. (c) Right- and left-period-1 limit cycles with large size. (d) Right- and left-multiperiod limit cycles. (e) Right- and left-chaotic spiral attractors. (f) Right- and left-half-baked double-scroll chaotic attractors. (g) Standard doublescroll chaotic attractor. (h) Unbounded orbit. 
51607013, and 11602035 and the Natural Science Foundations of Jiangsu Province, China, under Grant no. BK20160282.

\section{References}

[1] C. N. Ngonghala, U. Feudel, and K. Showalter, "Extreme multistability in a chemical model system," Physical Review E: Statistical, Nonlinear, and Soft Matter Physics, vol. 83, no. 5, Article ID 056206, 2011.

[2] M. S. Patel, U. Patel, A. Sen et al., "Experimental observation of extreme multistability in an electronic system of two coupled Rössler oscillators," Physical Review E: Statistical, Nonlinear, and Soft Matter Physics, vol. 89, no. 2, Article ID 022918, 2014.

[3] C. Hens, S. K. Dana, and U. Feudel, "Extreme multistability: Attractor manipulation and robustness," Chaos: An Interdisciplinary Journal of Nonlinear Science, vol. 25, no. 5, Article ID 053112, 2015.

[4] F. Yuan, G. Wang, P. Jin, X. Wang, and G. Ma, "Chaos in a Meminductor-Based Circuit," International Journal of Bifurcation and Chaos, vol. 26, no. 8, Article ID 1650130, 2016.

[5] B.-C. Bao, Q. Xu, H. Bao, and M. Chen, "Extreme multistability in a memristive circuit," IEEE Electronics Letters, vol. 52, no. 12, pp. 1008-1010, 2016.

[6] B. Bao, T. Jiang, Q. Xu, M. Chen, H. Wu, and Y. Hu, "Coexisting infinitely many attractors in active band-pass filter-based memristive circuit," Nonlinear Dynamics, vol. 86, no. 3, pp. 1711-1723, 2016.

[7] B. C. Bao, H. Bao, N. Wang, M. Chen, and Q. Xu, "Hidden extreme multistability in memristive hyperchaotic system," Chaos, Solitons \& Fractals, vol. 94, pp. 102-111, 2017.

[8] H. Bao, N. Wang, B. Bao, M. Chen, P. Jin, and G. Wang, "Initial condition-dependent dynamics and transient period in memristor-based hypogenetic jerk system with four line equilibria," Communications in Nonlinear Science and Numerical Simulation, vol. 57, pp. 264-275, 2018.

[9] B. Bao, T. Jiang, G. Wang, P. Jin, H. Bao, and M. Chen, "Twomemristor-based Chua's hyperchaotic circuit with plane equilibrium and its extreme multistability," Nonlinear Dynamics, pp. $1-15,2017$.

[10] Z. Wang, A. Akgul, V.-T. Pham, and S. Jafari, "Chaos-based application of a novel no-equilibrium chaotic system with coexisting attractors," Nonlinear Dynamics, vol. 89, no. 3, pp. 1877-1887, 2017.

[11] S. Morfu, B. Nofiele, and P. Marquié, "On the use of multistability for image processing," Physics Letters A, vol. 367, no. 3, pp. 192-198, 2007.

[12] A. N. Pisarchik and U. Feudel, "Control of multistability," Physics Reports, vol. 540, no. 4, pp. 167-218, 2014.

[13] P. R. Sharma, M. D. Shrimali, A. Prasad, N. V. Kuznetsov, and G. A. Leonov, "Control of multistability in hidden attractors," The European Physical Journal Special Topics, vol. 224, no. 8, pp. 1485-1491, 2015.

[14] D. Dudkowski, A. Prasad, and T. Kapitaniak, "Perpetual Points: New Tool for Localization of Coexisting Attractors in Dynamical Systems," International Journal of Bifurcation and Chaos, vol. 27, no. 4, Article ID 1750063, 2017.

[15] Z. T. Njitacke, J. Kengne, H. B. Fotsin, A. N. Negou, and D. Tchiotsop, "Coexistence of multiple attractors and crisis route to chaos in a novel memristive diode bidge-based Jerk circuit," Chaos, Solitons \& Fractals, vol. 91, pp. 180-197, 2016.
[16] J. Kengne, Z. N. Tabekoueng, V. K. Tamba, and A. N. Negou, "Periodicity, chaos, and multiple attractors in a memristorbased Shinriki's circuit," Chaos: An Interdisciplinary Journal of Nonlinear Science, vol. 25, no. 10, Article ID 103126, 2015.

[17] J. Kengne, Z. T. Njitacke, and H. B. Fotsin, "Dynamical analysis of a simple autonomous jerk system with multiple attractors," Nonlinear Dynamics, 2015.

[18] B. C. Bao, Q. D. Li, N. Wang, and Q. Xu, "Multistability in Chua's circuit with two stable node-foci," Chaos: An Interdisciplinary Journal of Nonlinear Science, vol. 26, no. 4, Article ID 043111, 2016.

[19] M. Chen, Q. Xu, Y. Lin, and B. Bao, "Multistability induced by two symmetric stable node-foci in modified canonical Chua's circuit," Nonlinear Dynamics, vol. 87, no. 2, pp. 789-802, 2017.

[20] J. Kengne, A. N. Negou, and D. Tchiotsop, "Antimonotonicity, chaos and multiple attractors in a novel autonomous memristor-based jerk circuit," Nonlinear Dynamics, vol. 88, no. 4, pp. 2589-2608, 2017.

[21] B. Bao, H. Qian, Q. Xu, M. Chen, J. Wang, and Y. Yu, "Coexisting behaviors of asymmetric attractors in hyperbolictype memristor based hopfield neural network," Frontiers in Computational Neuroscience, vol. 11, no. 81, pp. 1-14, 2017.

[22] J. C. Sprott, S. Jafari, A. J. M. Khalaf, and T. Kapitaniak, "Megastability: Coexistence of a countable infinity of nested attractors in a periodically-forced oscillator with spatiallyperiodic damping," The European Physical Journal Special Topics, vol. 226, no. 9, pp. 1979-1985, 2017.

[23] E. B. Ngouonkadi, H. B. Fotsin, P. Louodop Fotso, V. Kamdoum Tamba, and H. A. Cerdeira, "Bifurcations and multistability in the extended Hindmarsh-Rose neuronal oscillator," Chaos, Solitons \& Fractals, vol. 85, pp. 151-163, 2016.

[24] C. Li and J. C. Sprott, "Multistability in the lorenz system: A broken butterfly," International Journal of Bifurcation and Chaos, vol. 24, no. 10, Article ID 1450131, 2014.

[25] Q. Xu, Y. Lin, B. Bao, and M. Chen, "Multiple attractors in a nonideal active voltage-controlled memristor based Chua's circuit," Chaos, Solitons \& Fractals, vol. 83, pp. 186-200, 2016.

[26] O. S. Ojoniyi and A. N. Njah, "A 5D hyperchaotic Sprott B system with coexisting hidden attractors," Chaos, Solitons \& Fractals, vol. 87, pp. 172-181, 2016.

[27] G. Huang and J. Cao, "Multistability of neural networks with discontinuous activation function," Communications in Nonlinear Science and Numerical Simulation, vol. 13, no. 10, pp. 22792289, 2008.

[28] T. Malashchenko, A. Shilnikov, and G. Cymbalyuk, "Six types of multistability in a Neuronal model based on slow calcium current," PLoS ONE, vol. 6, no. 7, Article ID e21782, 2011.

[29] F. Corinto and M. Forti, "Memristor circuits: flux-charge analysis method," IEEE Transactions on Circuits and Systems I: Regular Papers, vol. 63, no. 11, pp. 1997-2009, 2016.

[30] F. Corinto and M. Forti, "Memristor Circuits: Bifurcations without Parameters," IEEE Transactions on Circuits and Systems I: Regular Papers, vol. 64, no. 6, pp. 1540-1551, 2017.

[31] B.-C. Bao, "Reply: Comment on 'Is memristor a dynamic element?"' IEEE Electronics Letters, vol. 50, no. 19, pp. 1344-1345, 2014.

[32] M. Itoh and L. O. Chua, "Duality of memristor circuits," International Journal of Bifurcation and Chaos, vol. 23, no. 1, Article ID 1330001, 2013.

[33] F. Z. Wang, "A triangular periodic table of elementary circuit elements," IEEE Transactions on Circuits and Systems I: Regular Papers, vol. 60, no. 3, pp. 616-623, 2013. 
[34] B. Bao, F. Hu, Z. Liu, and J. Xu, "Mapping equivalent approach to analysis and realization of memristor-based dynamical circuit," Chinese Physics B, vol. 23, no. 7, Article ID 070503, 2014.

[35] A. L. Fitch, D. Yu, H. H. C. Iu, and V. Sreeram, "Hyperchaos in a memristor-based modified canonical Chua's circuit," International Journal of Bifurcation and Chaos, vol. 22, no. 6, Article ID 1250133, 2012.

[36] F. Yuan, G. Wang, and X. Wang, "Chaotic oscillator containing memcapacitor and meminductor and its dimensionality reduction analysis," Chaos: An Interdisciplinary Journal of Nonlinear Science, vol. 27, no. 3, Article ID 033103, 2017.

[37] J. Ma, F. Wu, G. Ren, and J. Tang, "A class of initials-dependent dynamical systems," Applied Mathematics and Computation, vol. 298, pp. 65-76, 2017.

[38] G. A. Leonov, N. V. Kuznetsov, and T. N. Mokaev, "Hidden attractor and homoclinic orbit in Lorenz-like system describing convective fluid motion in rotating cavity," Communications in Nonlinear Science and Numerical Simulation, vol. 28, no. 1-3, pp. 166-174, 2015.

[39] V. T. Pham, S. Vaidyanathan, C. K. Volos, and S. Jafari, "Hidden attractors in a chaotic system with an exponential nonlinear term," The European Physical Journal Special Topics, vol. 224, no. 8, Article ID A1507, pp. 1507-1517, 2015.

[40] D. Dudkowski, S. Jafari, T. Kapitaniak, N. V. Kuznetsov, G. A. Leonov, and A. Prasad, "Hidden attractors in dynamical systems," Physics Reports, vol. 637, pp. 1-50, 2016.

[41] S. Jafari, J. C. Sprott, V.-T. Pham, C. Volos, and C. Li, "Simple chaotic 3D flows with surfaces of equilibria," Nonlinear Dynamics, vol. 86, no. 2, pp. 1349-1358, 2016.

[42] V.-T. Pham, S. Jafari, C. Volos, A. Giakoumis, S. Vaidyanathan, and T. Kapitaniak, "A chaotic system with equilibria located on the rounded square loop and its circuit implementation," IEEE Transactions on Circuits and Systems II: Express Briefs, vol. 63, no. 9, pp. 878-882, 2016.

[43] V.-T. Pham, S. Jafari, X. Wang, and J. Ma, "A chaotic system with different shapes of equilibria," International Journal of Bifurcation and Chaos, vol. 26, no. 4, Article ID 1650069, 2016.

[44] N. V. Kuznetsov, G. A. Leonov, M. V. Yuldashev, and R. V. Yuldashev, "Hidden attractors in dynamical models of phaselocked loop circuits: limitations of simulation in MATLAB and SPICE," Communications in Nonlinear Science and Numerical Simulation, vol. 51, pp. 39-49, 2017.

[45] Q. Li, S. Hu, S. Tang, and G. Zeng, "Hyperchaos and horseshoe in a $4 \mathrm{D}$ memristive system with a line of equilibria and its implementation," International Journal of Circuit Theory and Applications, vol. 42, no. 11, pp. 1172-1188, 2014. 


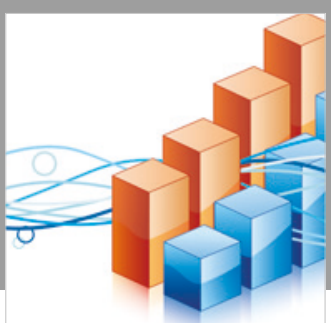

Advances in

Operations Research

\section{-n-m}
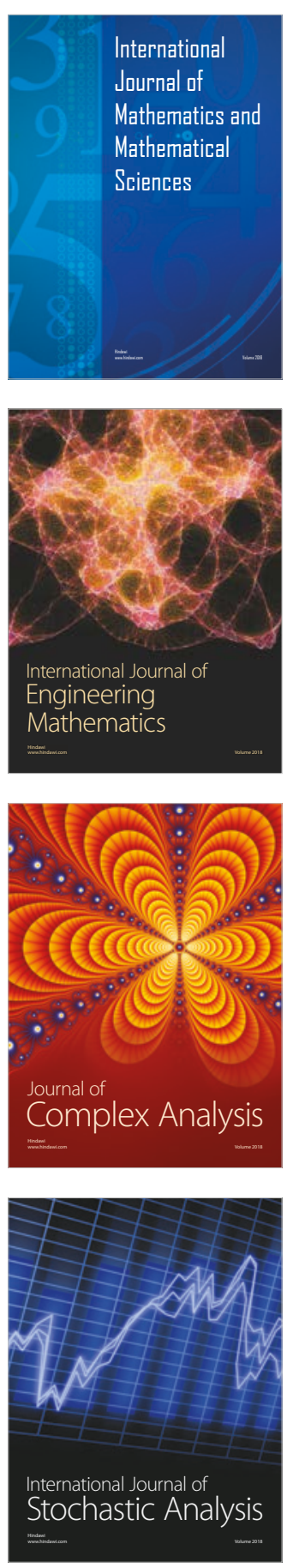
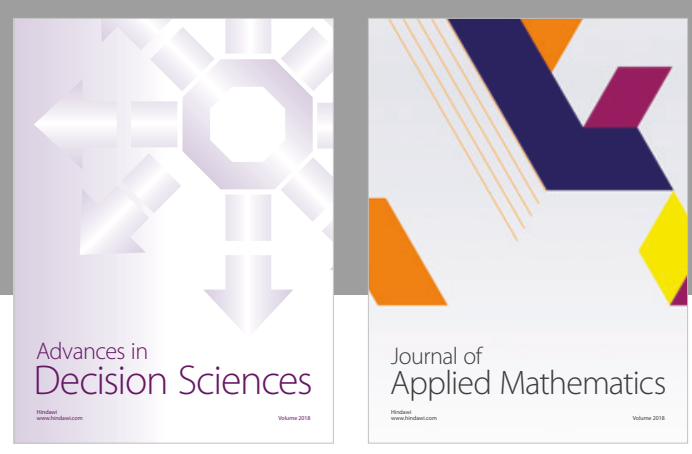

Journal of

Applied Mathematics
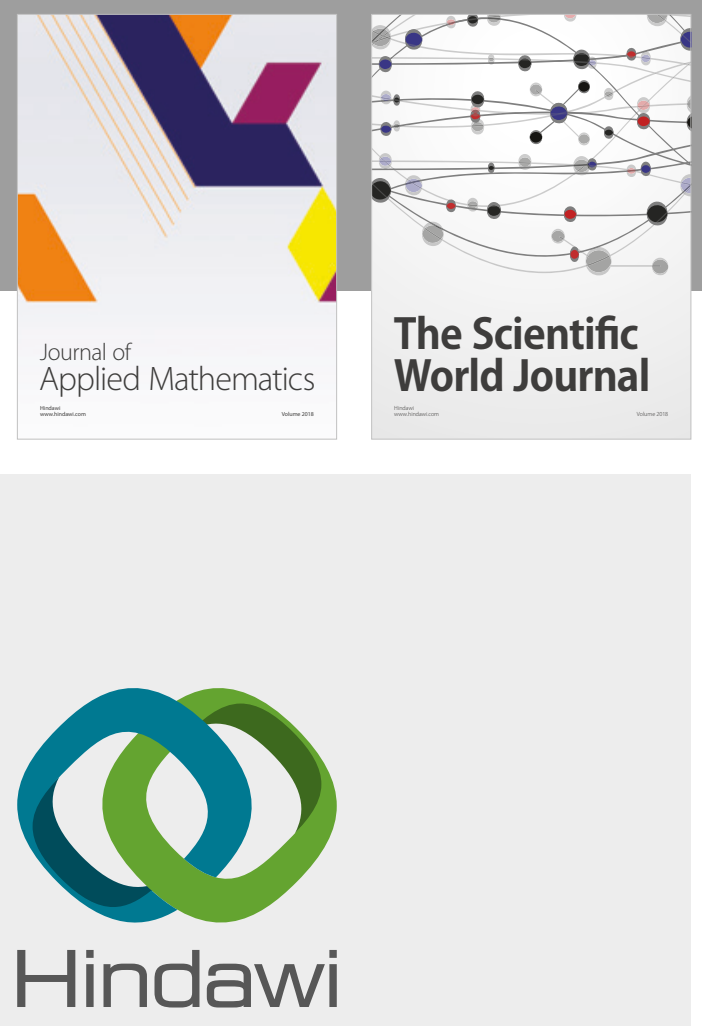

Submit your manuscripts at

www.hindawi.com

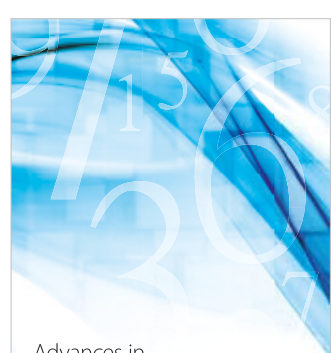

Advances in
Numerical Analysis
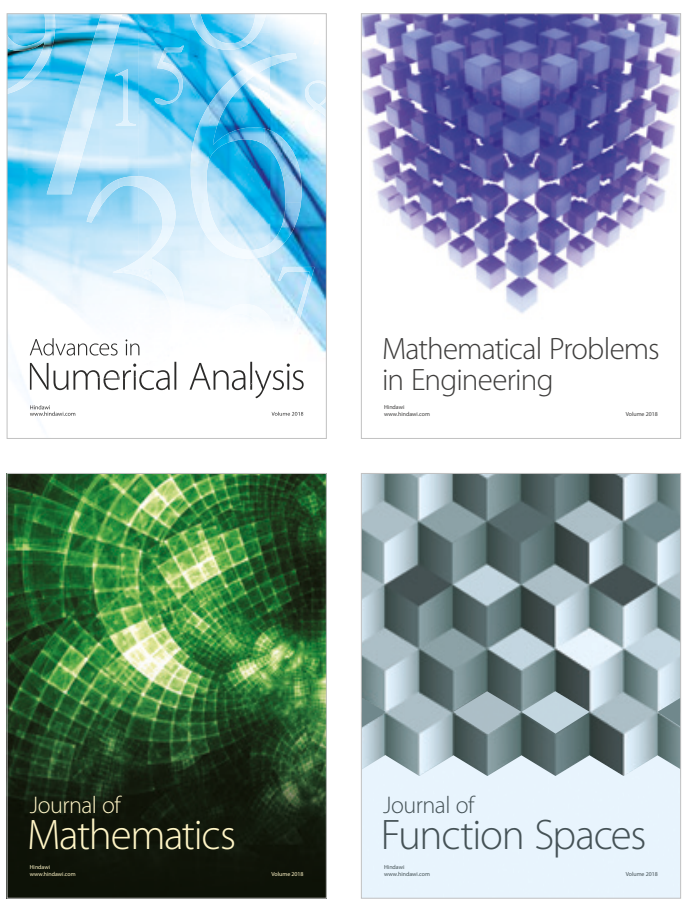

Mathematical Problems in Engineering

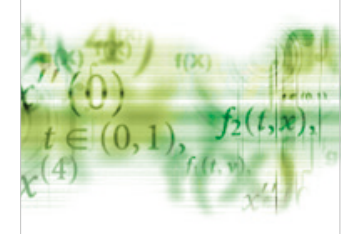

International Journal of

Differential Equations

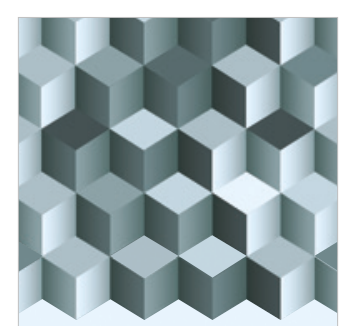

Journal of

Function Spaces
The Scientific

World Journal

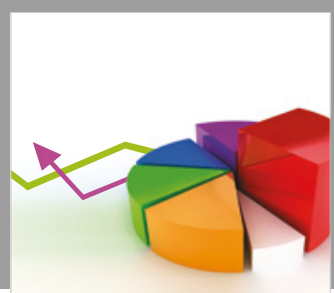

Journal of

Probability and Statistics
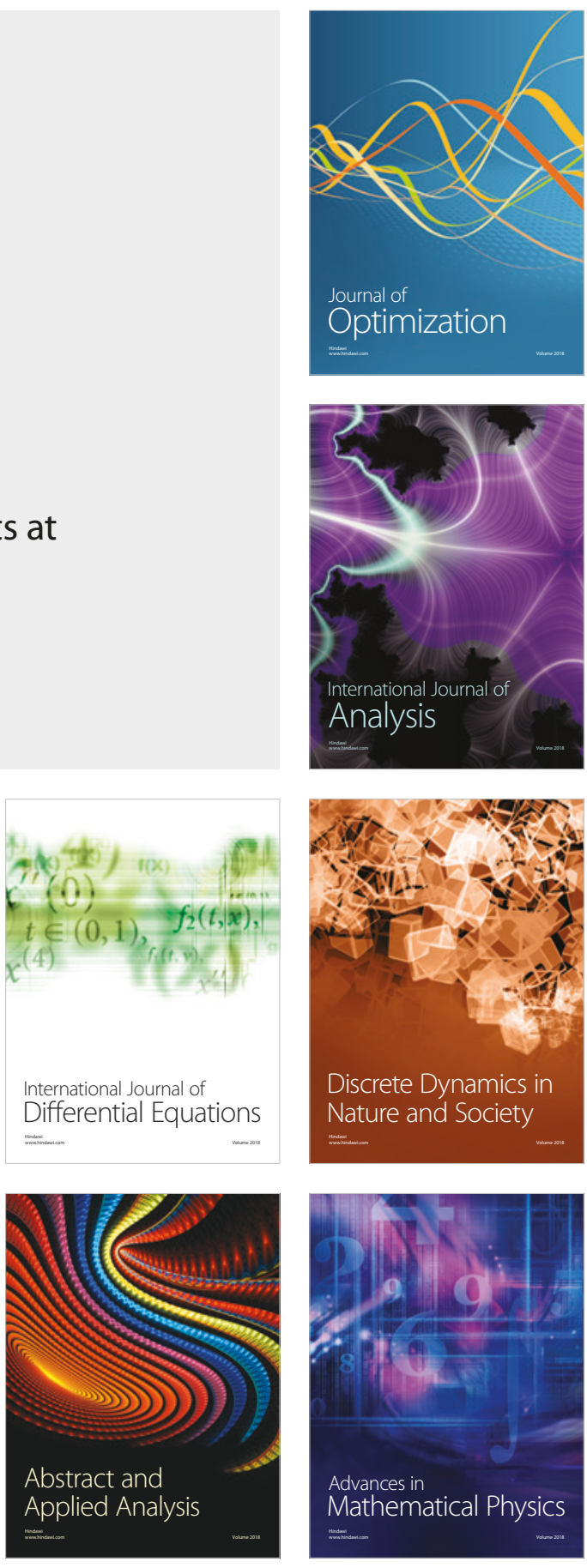